\title{
Needs Assessment for an Infant and Toddler Food Allergy Curriculum for Pediatric Residents
}

\author{
Marielle Young ${ }^{1}$, Ian Roy ${ }^{1}$, Ariel Frey-Vogel ${ }^{1}$, Kristina Dzara ${ }^{1}$, and Michael Pistiner ${ }^{1}$ \\ ${ }^{1}$ Massachusetts General Hospital
}

May 12, 2021

Conflicts of Interest: Marielle C. Young, Ian R. Roy, Ariel S. Frey-Vogel, and Kristina Dzara report no conflicts of interest. Michael Pistiner has served on advisory boards of kaléo, DBV Technologies, and Novartis, has received funding from kaléo, DBV Technologies, and the National Peanut Board, and is a co-founder of AllergyHome and of Allergy Certified Training.

Financial Support: This project was completed without financial support.

Keywords: food allergy, anaphylaxis, infant allergy, toddler allergy, pediatric residents, residency needs assessment, residency curriculum

Main Text:

To the Editor,

The prevalence of food allergy (FA) is increasing in the United States ${ }^{1}$ and it is estimated that 8-10\% of all children and $6 \%$ of children between 0 and 2 years of age have a FA. ${ }^{2,3}$ Recent publications have highlighted clinical practice deficits, including the inappropriate treatment of anaphylaxis ${ }^{4}$ and the lack of implementation of peanut feeding guidelines by primary care clinicians. ${ }^{5}$ Although pediatricians are frontline providers for infants ( $<12$ months) and toddlers (12-35 months) with FA, the Accreditation Council for Graduate Medical Education (ACGME) does not require a pediatric allergy curriculum as part of pediatric residency training.

We set out to explore the educational needs, attitudes, and satisfaction of the pediatric residents (PR) at MassGeneral Hospital for Children ( $\mathrm{MGH} f \mathrm{C}$ ) in Boston, MA. Our main objective was to investigate sources of information PR use to gain knowledge about infant/toddler FA and their comfort evaluating, diagnosing, treating, managing, and counseling patients and their families regarding infant/toddler FA and anaphylaxis.

A needs assessment survey was developed by pediatric clinicians, including a pediatric allergist. The survey instrument consisted of twelve questions exploring the following topics: FA training prior to residency, resources utilized for information, comfort evaluating and managing FA, comfort diagnosing and treating anaphylaxis, comfort counseling families about a variety of FA topics, satisfaction with current resident training opportunities (if present), and level of postgraduate training. Using best practices in survey design, cognitive interviewing was performed with a panel of pediatric clinicians to ensure face validity and the survey was updated iteratively to incorporate recommended changes. The final survey instrument was deemed to meaningfully cover the appropriate topics, to be easy to understand, and to be of manageable length.

The anonymous survey was administered via Research Electronic Data Capture (REDCap). A survey link and three follow-up emails were sent to the population of 64 pediatric and pediatric/internal medicine residents at MGH $f$ C. The study was exempted by the MassGeneral Brigham Human Research Office. Results are presented using descriptive and inferential statistics. Analyses were conducted in Stata/IC Version 16.1 
(StataCorp: College Station, Texas) and two-sided $p$ - values of less than $\alpha=0.05$ were considered statistically significant.

A total of 51 PR completed the needs assessment survey, representing a $79.7 \%$ response rate and a $100.0 \%$ completion rate. $37.3 \%$ (19) of the respondents were postgraduate year 1 (PGY-1), 29.4\% (15) were PGY-2, $27.4 \%$ (14) were PGY-3, and $5.9 \%$ (3) were PGY-4.

Prior to residency entry, only $5.9 \%$ of $\mathrm{PR}$ received formal training regarding infant/toddler FA. During residency, 31.4\% (16) received lectures, workshops, or simulations and 70.6\% (36) reported feeling dissatisfied with the current resident training opportunities concerning this topic.

The most commonly reported sources for information regarding infant/toddler FA and anaphylaxis included outpatient pediatric clinicians (i.e., community pediatricians) $(72.5 \%, 37)$, other pediatric residents $(58.8 \%$, $30)$, and Emergency Department clinicians $(52.9 \%, 28)$. However, when asked about their single most informative resource, outpatient pediatric clinicians $(29.4 \%, 15)$, allergy electives $(15.7 \%, 8)$, and allergytrained clinicians (i.e., staff allergists) $(15.7 \%, 8)$ were reported in that order (Figure 1).

The majority of PR felt uncomfortable evaluating $(76.5 \%, 39)$ and managing $(86.3 \%, 44)$ infant/toddler FA. However, 68.6\% (35) of PR felt comfortable diagnosing and treating infant/toddler anaphylaxis, respectively.

When examining factors that may contribute to PR's clinical comfort, we found a strong association between formal engagement with allergy-trained clinicians, defined as the completion of an allergy elective and/or the utilization of staff allergy-trained clinicians when in need of allergy-related advice, and comfort treating infant/toddler anaphylaxis. After adjusting for years of postgraduate training, PR who had formal engagement with allergy-trained clinicians had 8.27 times the odds $(\mathrm{OR}=8.27 ; 95 \%$ C.I. 1.16-59.01; $p$-value $=$ 0.035) of feeling comfortable treating infant/toddler anaphylaxis as compared to PR who did not.

Our study identified several educational deficiencies, including a lack of, and dissatisfaction with, training opportunities and a lack of comfort evaluating, managing, and counseling families about FA topics. However, we also showed that formal engagement with allergy-trained clinicians was strongly associated with increased comfort treating anaphylaxis.

This study demonstrates several gaps regarding PR's sources of information and comfort with infant/toddler FA and anaphylaxis. The majority of PR enter residency without training in infant/toddler FA and anaphylaxis. Residents then, in the absence of a standard allergy curriculum, seek information about infant/toddler FA and anaphylaxis from multiple sources - many of whom are not allergists. When presented with lectures, workshops, and simulation experiences about FA, PR are largely dissatisfied with them. Furthermore, the top three resources utilized for information about infant/toddler FA and anaphylaxis did not include allergy experiences, despite PR reporting that two of the top three single most informative resources were allergy electives and allergy-trained clinicians. Overall, these findings suggest that the design and implementation of a standard curriculum, while emphasizing engagement with pediatric allergists, may present improved educational opportunities.

This study was designed to be descriptive; therefore, we did not conduct a power analysis and sample size calculation prospectively. However, this analysis illuminates the need for an allergy medicine curriculum emphasizing engagement with allergy-trained clinicians among pediatric residents at MGH $f \mathrm{C}$. While not generalizable beyond our setting, we have been successfully funded by The Allergists' Foundation of the American College of Allergy, Asthma, \& Immunology to expand this educational needs assessment nationally to determine if the findings at our institution are generalizable to pediatric residents as a whole within the United States. Furthermore, this needs assessment purposefully focused on attitudes, satisfaction, and comfort measured through self-report. Future studies might focus on direct measures of residents' clinical proficiency and how PR apply FA and anaphylaxis knowledge on their own.

This study is the first to examine pediatric residents' knowledge sources and comfort evaluating, diagnosing, treating, managing, and counseling families regarding infant/toddler FA and anaphylaxis and adds to the literature in several ways. First, we examined a population of pediatric and pediatric/internal medicine 
residents, whereas the existing literature has focused on other populations. ${ }^{6-8}$ Second, we explored where PR seek information about infant/toddler FA and anaphylaxis. These findings support the need for a national needs assessment which may guide the design and implementation of a standard pediatric allergy curriculum for all pediatric residency programs.

Acknowledgements: We are grateful to the MassGeneral Hospitalfor Children pediatric residents who voluntarily participated in this study. We also thank Mharlove Andre for her support during the preparation of this manuscript.

Impact Statement: Although pediatric residents are frontline providers for infants and toddlers with food allergy, they may lack comfort evaluating, managing, and counseling families about food allergy topics. Formal engagement with allergy-trained clinicians may improve pediatric residents' comfort treating allergic conditions.

\section{References:}

1. Sicherer SH and Sampson HA. Food Allergy: A review and update on epidemiology, pathogenesis, diagnosis, prevention and management. J Allergy Clin Immunol. 2018;141(1):41-58.

2. Sicherer SH, Muñoz-Furlong A, Godbold JH, Sampson HA. US prevalence of self-reported peanut, tree nut, and sesame allergy: 11-year follow-up. J Allergy Clin Immunol. 2010;125(6):1322-6.

3. American Academy of Allergy Asthma and Immunology. (2020, September). Allergy Statistics. https://www.aaaai.org/about-aaaai/newsroom/allergy-statistics

4. Michelson KA, Dribin TE, Vyles D, Neuman MI. Trends in emergency care for anaphylaxis. J Allergy Clin Immunol Pract. 2020;8(2):767-768.e2.

5. Gupti, RS, Bilaver RA, Johnson JL, et al. Assessment of Pediatrician Awareness and Implementation of the Addendum Guidelines for the Prevention of Peanut Allergy in the United States. JAMA Netw Open. 2020;3(7):e2010511.

6. Lanser BJ, Covar R, Bird, JA. Food allergy needs assessment, training curriculum and knowledge assessment for child care. Ann Allergy Asthma Immunol. 2016;116(6):533-537.e4.

7. Carlisle SK, Vargas PA, Noone S, et al. Food Allergy Education for School Nurses: A Needs Assessment Survey by the Consortium of Food Allergy Research. J Sch Nurse. 2010;26(5):360-367.

8. Vargas PA, Sicherer SH, Christine L, et al. Developing a food allergy curriculum for parents. Pediatric Allergy Immunol. 2011;22(6):575-582.

Table 1. The unadjusted and adjusted independent associations between the predictor variable, formal engagement with allergy-trained clinicians, and four mutually exclusive clinical comfort outcome variables.

\section{Figure Legends}

Figure 1. The number of pediatric residents who have utilized each resource to gain knowledge and experience regarding infant/toddler food allergy and anaphylaxis (light blue) and the number of pediatric residents who believe that each resource is the single most informative regarding infant/toddler food allergy and anaphylaxis (dark blue). 


\begin{tabular}{|c|c|c|c|c|c|c|}
\hline & \multicolumn{3}{|c|}{ Unadjusted Univariate Logistic Regression } & \multicolumn{3}{|c|}{ Adjusted* Multivariate Logistic Regression } \\
\hline & Odds Ratio (OR) & $95 \%$ C.I. & $p$-value & Odds Ratio (OR) & $95 \%$ C.I. & $p$-value \\
\hline \multirow{2}{*}{\multicolumn{7}{|c|}{$\begin{array}{l}\text { Outcome Variable: Comfort Evaluating Infant/Toddler Food Allergy } \\
\text { Engagement with Allergy }\end{array}$}} \\
\hline & & & & & & \\
\hline Yes & 7.64 & 1.74 to 33.58 & 0.007 & 5.13 & 0.85 to 30.95 & 0.075 \\
\hline No & 1.00 & $\operatorname{Ref}$ & & 1.00 & Ref & \\
\hline \multicolumn{7}{|c|}{ Outcome Variable: Comfort Managing Infant/Toddler Food Allergy } \\
\hline \multicolumn{7}{|c|}{ Engagement with Allergy } \\
\hline Yes & 4.83 & 0.84 to 27.93 & 0.078 & 2.12 & 0.25 to 17.78 & 0.487 \\
\hline No & 1.00 & Ref & & 1.00 & Ref & \\
\hline \multirow{2}{*}{\multicolumn{7}{|c|}{$\begin{array}{l}\text { Outcome Variable: Comfort Diagnosing Infant/Toddler Anaphylaxis } \\
\text { Engagement with Allergy }\end{array}$}} \\
\hline & & & & & & \\
\hline Yes & 2.53 & 0.68 to 9.39 & 0.166 & 1.23 & 0.26 to 5.91 & 0.795 \\
\hline No & 1.00 & Ref & & 1.00 & Ref & \\
\hline \multicolumn{7}{|c|}{ Outcome Variable: Comfort Treating Infant/Toddler Anaphylaxis } \\
\hline \multicolumn{7}{|c|}{ Engagement with Allergy } \\
\hline Yes & 7.41 & 1.46 to 37.57 & 0.016 & 8.27 & 1.16 to 59.01 & 0.035 \\
\hline No & 1.00 & Ref & & 1.00 & Ref & \\
\hline
\end{tabular}


Figure 1. Infant and Toddler Food Allergy and Anaphylaxis Resources Utilized by Pediatric Residents

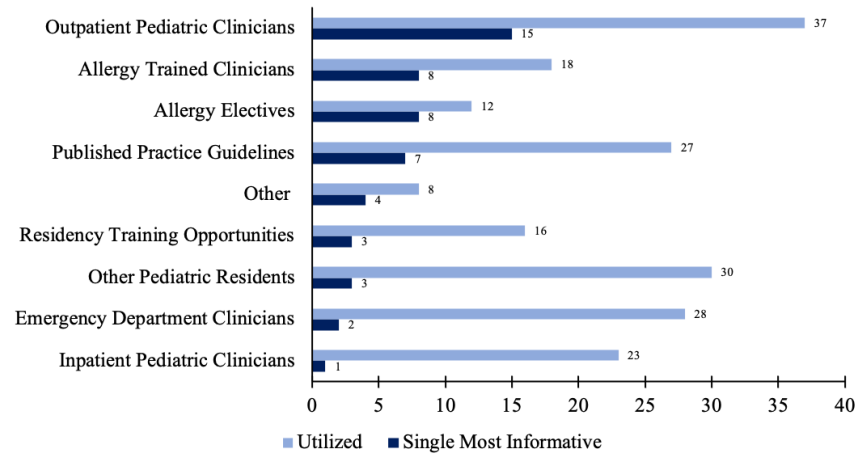

